\title{
Epidemiology of Meningitis in an HIV-Infected Ugandan Cohort
}

\author{
Radha Rajasingham,* Joshua Rhein, Kate Klammer, Abdu Musubire, Henry Nabeta, Andrew Akampurira, Eric C. Mossel, \\ Darlisha A. Williams, Dave J. Boxrud, Mary B. Crabtree, Barry R. Miller, Melissa A. Rolfes, Supatida Tengsupakul, \\ Alfred O. Andama, David B. Meya, and David R. Boulware \\ University of Minnesota, Minneapolis, Minnesota; Infectious Disease Institute, Makerere University, Kampala, Uganda; \\ Minnesota Department of Health, St. Paul, Minnesota; Centers for Disease Control and Prevention, Fort Collins, Colorado; \\ Department of Medicine, Makerere University College of Health Sciences, Kampala, Uganda
}

\begin{abstract}
There is limited understanding of the epidemiology of meningitis among human immunodeficiency virus (HIV)-infected populations in sub-Saharan Africa. We conducted a prospective cohort study of HIV-infected adults with suspected meningitis in Uganda, to comprehensively evaluate the etiologies of meningitis. Intensive cerebrospiral fluid (CSF) testing was performed to evaluate for bacterial, viral, fungal, and mycobacterial etiologies, including neurosyphilis,16s ribosomal DNA (rDNA) polymerase chain reaction (PCR) for bacteria, Plex-ID broad viral assay, quantitative-PCR for HSV-1/2, cytomegalovirus (CMV), Epstein-Barr virus (EBV), and Toxoplasma gondii; reverse transcription-PCR (RT-PCR) for Enteroviruses and arboviruses, and Xpert MTB/RIF assay. Cryptococcal meningitis accounted for $60 \%$ (188 of 314) of all causes of meningitis. Of 117 samples sent for viral PCR, 36\% were EBV positive. Among cryptococcal antigen negative patients, the yield of Xpert MTB/RIF assay was 22\% (8 of 36). After exclusion of cryptococcosis and bacterial meningitis, 61\% (43 of 71) with an abnormal CSF profile had no definitive diagnosis. Exploration of new TB diagnostics and diagnostic algorithms for evaluation of meningitis in resource-limited settings remains needed, and implementation of cryptococcal diagnostics is critical.
\end{abstract}

\section{INTRODUCTION}

Meningitis is a common cause of death in Africa. Historically in the African meningitis belt, bacterial pathogens such as Neisseria meningitidis and Streptococcus pneumoniae have been the most common etiologies, resulting in an estimated 800,000 cases between 1996 and 2010. ${ }^{1}$ During non-epidemic situations in the African meningitis belt, surveillance conducted in Burkina Faso, Ghana, Niger, and Mali estimate the most common etiological agents of meningitis are Neisseria meningitidis (37-52\%), Streptococcus pneumoniae (27-43\%), and Haemophilus influenzae (5-31\%). ${ }^{2}$ Because of these findings, the World Health Organization (WHO) emphasizes empiric ceftriaxone as the first-line therapy, ${ }^{2}$ despite the geographic diversity of the continent.

The higher prevalence of human immunodeficiency virus (HIV) infection in Eastern and Southern Africa has a dramatic impact on the etiologies of meningitis. In Malawi, South Africa, Uganda, Zambia, and Zimbabwe, cryptococcal meningitis is the predominant etiology of meningitis followed by tuberculosis (TB) meningitis (TBM) in adults. ${ }^{3-8}$ In both South Africa and Malawi, Cryptococcus is more common than all causes of bacterial meningitis combined. ${ }^{9,10}$ In Ethiopia, the most common neurologic manifestation has been thought to be cerebral toxoplasmosis, followed by TBM and Cryptococcus, ${ }^{11}$ although recent studies suggest a very large burden of cryptococcosis among those with advanced HIV. ${ }^{12,13}$

Unfortunately, in many African countries, outside of a research setting, microbiological diagnoses of meningitis are difficult to ascertain, and patients are treated empiricallyoften initially with antibacterial agents per WHO guidance. ${ }^{2}$ Even in the setting of well-resourced research studies, microbiological meningitis diagnoses are made in a minority of cases. $^{3,14,15}$ TBM is notoriously difficult to diagnose with

* Address correspondence to Radha Rajasingham, 420 Delaware Street, MMC250, Minneapolis, MN 55455. E-mail: Radha.Rajasingham@ gmail.com widely differing data on the use of microscopy for acid fast bacilli (AFB) smear on CSF. ${ }^{16-19}$ Mycobacterial culture takes up to 6 weeks to grow, making culture obsolete for guiding clinical management. CSF interferon-gamma (IFN- $\gamma$ ) levels have been suggested as a TBM diagnostic biomarker, yet thresholds vary. ${ }^{20,21}$ The debut of the Xpert MTB/RIF assay, an automated polymerase chain reaction (PCR) molecular assay, is encouraging. The assay is relatively rapid (results in $\sim 2.5$ hours) and has shown excellent performance with sputum for pulmonary TB (i.e., sensitivity $86 \%$, specificity $>95 \%$ ). ${ }^{22}$ Currently, performance data of the Xpert MTB/RIF in CSF suggest a more limited sensitivity $(\sim 60 \%))^{23,24}$

This study aimed to comprehensively evaluate the microbiologic etiologies of meningitis in an HIV-infected inpatient adult population with suspected meningitis in Kampala, Uganda.

\section{METHODS}

Setting and patients. We conducted a prospective cohort study of HIV-infected adult inpatients consecutively presenting with suspected meningitis between November 2010 and October 2012 at Mulago National Hospital-a tertiary referral hospital in Kampala, Uganda, as part of screening for the Cryptococcal Optimal ART Timing (COAT) trial. ${ }^{25} \mathrm{We}$ included HIV-infected patients, not receiving anti-retroviral therapy (ART) presenting with suspected meningitis, and gave written informed consent. Persons known to be receiving ART were excluded as immune reconstitution inflammatory syndrome (IRIS) creates different diagnostic considerations beyond the scope of our aims. ${ }^{26}$ Clinical suspicion for meningitis was based on one or more symptoms of headache, fever, neck stiffness, or altered mental status. The CSF was obtained for routine examination and microbiological testing, as described below. If HIV-serostatus was unknown, rapid HIV testing was performed. If clinically indicated chest radiograph and sputum examination were obtained. Neuroradiology was obtained if patients had focal neurologic deficits.

Routine CSF examination. Prospectively, the Makerere Microbiology Laboratory performed CSF testing of white 
cell count, protein, and India ink. CSF glucose testing was unavailable. Cryptococcal antigen by latex agglutination was performed by the Makerere University Johns Hopkins University laboratory. After centrifugation $(3000 \times g \times 10$ minutes $)$ of $\geq 5 \mathrm{~mL}$ initial CSF volume, Gram stain, India ink, and acid fast bacilli stain were performed on the concentrated pellet and the CSF supernatant was frozen at $-80^{\circ} \mathrm{C}$ for later testing. Additionally, fungal and bacterial CSF cultures were performed; however, these later culture results did not influence TBM testing. Mycobacterial culture was unavailable. Cryptococcal meningitis was diagnosed by CSF culture or positive CSF cryptococcal antigen by latex agglutination or lateral flow assay (Immy Inc., Norman, OK). Lateral flow assay was prospectively used beginning in $2011 .^{27}$ Definitive bacterial meningitis was diagnosed by CSF culture, Gram stain, or 16 s rDNA sequencing.

Further diagnostic studies. Additional CSF testing was performed on 63 samples negative for cryptococcosis to determine the etiology of meningitis, and a subset of 54 samples with Cryptococcus to identify potential concurrent infections. Samples were selected based on available remaining CSF for further testing. 16s rDNA amplification was performed at the University of Minnesota to detect potential culture-negative bacterial meningitis (Supplemental Methods). At the Minnesota Department of Health (MDH), quantitative-PCR was performed for herpes simplex (HSV-1/2), cytomegalovirus (CMV), Epstein-Barr virus (EBV), Toxoplasma gondii, and RT-PCR for enterovirus. Neurosyphilis testing was performed using venereal disease research laboratory (VDRL).

Additionally, Ibis PLEX-ID (Abbott, IL) Broad Viral 1 Assay was performed, per manufacturer's instructions, to detect herpesviruses, adenoviruses, parvovirus B19, enteroviruses, and polyomaviruses through a broad range mass spectrometry platform. The MDH tested specimens in parallel to evaluate the PLEX-ID system in comparison with established PCR/RT-PCR methods.

Arboviral meningoencephalitis evaluation was performed on 111 samples negative for cryptococcosis and TBM, in collaboration with Centers for Disease Control and Prevention (CDC), by culture and genus-specific PCR for alphavirus, orthobunyavirus, and flavivirus. Sixty-two serum samples with sufficient remaining sample were evaluated for immunoglobulin $\mathrm{M}$ (IgM) for West Nile, yellow fever, dengue, chikungunya, and Zika viruses.

Xpert MTB/RIF assay. In 2011-2012, Xpert MTB/RIF assay (Cepheid, Sunnyvale, CA) was performed in Uganda, according to manufacturer's instructions, on 63 CSF samples, at physician discretion, for patients with a lymphocytic pleocytosis to evaluate for TBM. The $1 \mathrm{~mL} \mathrm{CSF}$ was mixed with $1 \mathrm{~mL}$ of Xpert MTB/RIF sample reagent to a total volume of $2.0 \mathrm{~mL}$. Specimens were incubated at room temperature for 15 minutes with a manual vigorous shaking 10-20 times after 5-10 minutes. A minimum of $2 \mathrm{~mL}$ of specimen was transferred by sterile technique into the Xpert MTB/RIF cartridge and evaluated immediately. ${ }^{28}$

Cytokine evaluation. We additionally measured CSF cytokine levels, with specific interest in IFN- $\gamma$. Frozen CSF supernatant was shipped on dry ice $\left(-20^{\circ} \mathrm{C}\right)$ to the University of Minnesota. We measured CSF cytokines using a Luminex instrument and reagents, according to manufacturer's instructions (Bio-Rad, Hercules, CA). The IFN- $\gamma$ measurements in $\mathrm{pg} / \mathrm{mL}$ were converted to $\mathrm{IU} / \mathrm{mL}(40 \mathrm{pg} / \mathrm{mL}=1 \mathrm{IU} / \mathrm{mL})$.
Ethics statement. All participants (or a surrogate) provided written informed consent for lumbar puncture, CSF testing, CSF storage, and data collection. Institutional review board approval occurred at all institutions.

Data analysis and statistics. Statistical analysis was primarily descriptive with distributions characterized by median and interquartile range (IQR). A Fisher's exact test compared the frequency of EBV detection with and without a co-infection. Statistical analysis was conducted using SPSS version 22 (IBM, Armonk, NY).

\section{RESULTS}

Three hundred and fourteen consecutive HIV-infected patients presenting to Mulago Hospital in Kampala, Uganda with suspected meningitis were enrolled. Baseline demographic characteristics, along with characteristics of presentation are summarized in Table 1 . One hundred and eighty-eight (60\%) had confirmed cryptococcal meningitis by CSF culture or cryptococcal antigen (Figure 1). Of the remaining 126 patients, 8 were missing either CSF white blood cell (WBC) or protein information, and were excluded from the analysis given insufficient information. Forty-two (13.4\%) had a normal CSF WBC count $(<5$ cells $/ \mu \mathrm{L})$, normal CSF protein $(<45 \mathrm{mg} / \mathrm{dL})$, and cryptococcal negative. These 42 were classified as not having meningitis. Bacterial meningitis was present in five patients

TABLE 1

Baseline characteristics of $314 \mathrm{HIV}$-infected patients enrolled with suspected meningitis

\begin{tabular}{|c|c|c|}
\hline Characteristic & $\begin{array}{l}\text { Total cohort } \\
(N=314)\end{array}$ & $\begin{array}{l}\text { Cryptococcal meningitis } \\
(N=188)\end{array}$ \\
\hline $\begin{array}{l}\text { Age in years, median } \\
\text { (IQR) }\end{array}$ & 34 (IQR, 29 to 40$)$ & $35(\mathrm{IQR}, 30$ to 40$)$ \\
\hline Male Sex (\%) & $49 \%$ & $47 \%$ \\
\hline $\begin{array}{l}\mathrm{CD} 4 \text { cells/ } / \mu \mathrm{L} \\
\quad \text { median IQR)* }\end{array}$ & 41 (IQR, 7 to 69) & 17 (IQR, 7 to 70$)$ \\
\hline $\begin{array}{l}\text { Glasgow coma score, } \\
\text { median }\end{array}$ & $15(\mathrm{IQR}, 14$ to 15$)$ & $15(\mathrm{IQR}, 14$ to 15$)$ \\
\hline \multicolumn{3}{|l|}{ Subjective complaints } \\
\hline Headache & $290(92 \%)$ & $185(98 \%)$ \\
\hline Fever & $276(88 \%)$ & $165(88 \%)$ \\
\hline Neck pain & $224(71 \%)$ & $149(79 \%)$ \\
\hline General Weakness & $128(41 \%)$ & $76(40 \%)$ \\
\hline Vomiting & $114(36 \%)$ & $84(45 \%)$ \\
\hline Confusion & $109(35 \%)$ & $56(30 \%)$ \\
\hline Weight Loss & $98(31 \%)$ & $57(30 \%)$ \\
\hline Cough & $83(26 \%)$ & $45(24 \%)$ \\
\hline Seizures & $52(17 \%)$ & $30(16 \%)$ \\
\hline Photophobia & $45(14 \%)$ & $31(16 \%)$ \\
\hline Blurry Vision & $29(9 \%)$ & $21(11 \%)$ \\
\hline \multicolumn{3}{|l|}{$\begin{array}{l}\text { Outcomes at } \\
\text { hospital discharge }\end{array}$} \\
\hline Alive & $166(53 \%)$ & $111(59 \%)$ \\
\hline Dead & $84(27 \%)$ & $45(24 \%)$ \\
\hline Unknown & $64(20 \%)$ & $32(17 \%)$ \\
\hline \multicolumn{3}{|l|}{ CSF characteristics } \\
\hline $\begin{array}{l}\text { White cells/ } \mu \mathrm{L} \text {, } \\
\text { median }\end{array}$ & 4 (IQR, 4 to 90$)$ & 25 (IQR, 4 to 105$)$ \\
\hline $\begin{array}{l}\text { Protein mg/dL, } \\
\text { median }\end{array}$ & 79 (IQR, 39 to 174) & 91 (IQR, 41 to 173$)$ \\
\hline Percent & \multirow{2}{*}{$100 \%(\mathrm{IQR}, 81-100 \%)$} & $100 \%(\mathrm{IQR}, 85-100 \%)$ \\
\hline $\begin{array}{l}\text { Lymphocyte, } \\
\text { median }\end{array}$ & & \\
\hline
\end{tabular}




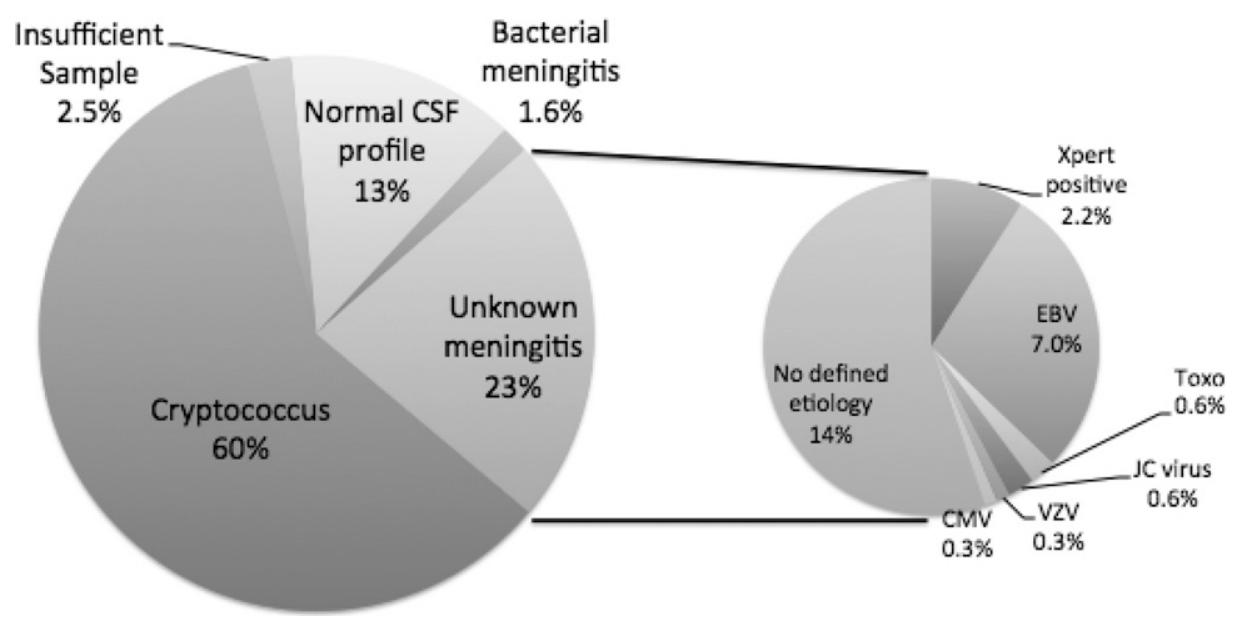

FIGURE 1. Etiologies of meningitis in Uganda among human immunodeficiency virus (HIV)-infected anti-retroviral therapy (ART)-naive persons. Cryptococcal meningitis accounted for the majority (60\%) of meningitis among hospitalized patients with suspected meningitis who received a lumbar puncture. Less common etiologies included: bacterial meningitis $(1.6 \%)$ and tuberculosis $(2.5 \%)$. Aseptic meningitis accounted for $23 \%$ (71 of 314) of etiologies overall. Extensive testing revealed testing positive for tuberculosis by GeneXpert $(N=8)$, EBV $(N=22)$, toxoplasmosis $(N=2)$, John Cunningham $(\mathrm{JC})$ virus $(N=2)$, VZV $(N=1)$, and CMV $(N=1)$. There were 43 specimens $(13.7 \%$ overall) with no etiology determined despite extensive molecular testing. The population was HIV-infected and not receiving HIV therapy. The percentages of the unknown meningitis sum to $>23 \%$ caused by multiple overlapping concurrent identification of viral pathogens. CMV = cytomegalovirus; $\mathrm{EBV}=$ Epstein-Barr virus; Toxo $=$ toxoplasmosis; Xpert $=$ GeneXpert MTB/RIF PCR assay. Insufficient samples were missing either cerebrospiral fluid (CSF) white blood cell (WBC) or protein results, most often caused by the CSF being grossly blood stained.

(1.6\%) with four positive Gram stains (S. pneumoniae $N=3$, Neisseria meningitidis) and one additional CSF sample with S. pneumoniae detected by $16 \mathrm{~s}$ PCR; bacterial cultures were negative in these samples.

The remaining 71 CSF samples (22.6\%) had either an elevated WBC count or elevated protein with a clinical diagnosis of meningitis of unknown etiology. The median age for this subgroup was 33 years (range 22 to 81 ) with $51 \%$ male. The median CD4 count was 29 cells/ $\mu \mathrm{L}$ (IQR, 6 to 48 ), with median Glasgow coma score of 14 (IQR, 12 to 15 ) and Karnofsky score of 40 (IQR, 40 to 50). Among these 71 persons of interest, the median CSF WBC count was 45 (IQR, 5 to135, max 880$)$ cells $/ \mu \mathrm{L}$ with a median lymphocyte percentage of $100 \%$ (IQR $81-100 \%$, min $52 \%$ ). Median CSF protein was 121 (IQR, 72-240, max 730) mg/dL, and CSF opening pressure was 18 (IQR, 13-29) $\mathrm{cmH}_{2} \mathrm{O}$ (normal $<20 \mathrm{cmH}_{2} \mathrm{O}$ ). The median CSF removed for diagnostics was 16 (IQR, 10-25) mL. Ziehl-Neelsen AFB stain was negative on all CSF samples.

Of the 71 patients with abnormal CSF of unknown etiology, $39 \%(N=29)$ survived to hospital discharge, $31 \%$ died $(N=$ $22), 4 \%(N=3)$ formally left the hospital against medical advice, and $24 \%(N=17)$ had unknown outcome.

Results from further diagnostics. One hundred and seventeen CSF samples (63 non-Cryptococcus, 54 Cryptococcus) were sent for further analysis through Toxoplasma gondii PCR, viral q-PCR/RT-PCR, and PLEX-ID (Table 2). Notably, $50 \%$ (58 of 117) of samples were negative for all tested viruses. The most prevalent virus isolated was EBV (36\%), with a similar prevalence in those with and without cryptococcal infection (39\% versus $33 \%$ respectively, $P=0.8$ ). Neurosyphilis was detected in $2.7 \%$ ( 3 of 111) of persons tested. Though testing for enterovirus, HSV-1 and HSV-2 was performed, there were no positive results by either PLEX-ID or RT-PCR.

Arboviral testing. Of 111 samples with sufficient CSF volume for arboviral testing, genus-specific RT-PCR for alphavirus, orthobunyavirus, and flavivirus were negative. In 62 serum specimens sent, IgM serologies were negative for West Nile, yellow fever, and chikungunya. The only non-negative serologies were for dengue ( $N=1$ positive, $N=2$ equivocal) and Zikavirus ( $N=1$ equivocal). Virus isolation by culture was negative on serum IgM positive and equivocal samples.

Xpert MTB/RIF assay results. Sixty-three CSF samples had sufficient CSF volume for further TB investigation among those with suspected TB meningitis. Of 36 patients with available demographic data, $64 \%$ were male with a median age of 35 years. Fifty of the CSF samples were run prospectively and 13 retrospectively on the GeneXpert platform. Eight CSF samples were found to be Xpert MTB/RIF positive $(12.7 \%$ of those tested), none of which were rifampin resistant. One sample was uninterpretable by Xpert. Twenty-seven of the $63 \mathrm{CSF}$ samples tested by GeneXpert were cryptococcal antigen positive. One sample was both cryptococcal antigen positive and Xpert MTB/RIF positive. Among cryptococcal-negative samples, 8 of 36 were positive for Xpert MTB/RIF for a prevalence of $22.2 \%$ (95\% confidence interval [CI]: 10-39\%).

TABLE 2

Results of molecular testing among persons with unknown or known etiology of meningitis

\begin{tabular}{lccc}
\hline \multicolumn{1}{c}{ Etiology } & $\begin{array}{c}\text { Total } \\
(N=117)\end{array}$ & $\begin{array}{c}\text { Cryptococcal meningitis } \\
(N=54)\end{array}$ & $\begin{array}{c}\text { TB meningitis by } \\
\text { GeneXpert }(N=4)\end{array}$ \\
\hline EBV & 42 & 21 & 3 \\
EBV + CMV & 3 & 2 & 0 \\
CMV & 2 & 0 & 0 \\
JC virus & 3 & 2 & 0 \\
Toxoplasma & 3 & 1 & 0 \\
EBV + JC virus & 3 & 2 & 1 \\
EBV + VZV & 1 & 0 & 0 \\
CMV + Toxoplasma & 1 & 0 & 0 \\
BK virus & 1 & 0 & 0 \\
Enterovirus & 0 & 0 & 0 \\
Negative & 58 & 26 & 0 \\
\hline
\end{tabular}

EBV = Epstein-Barr virus; CMV = cytomegalovirus; JC virus = John Cunningham virus; $\mathrm{VZV}=$ varicella zoster virus. 
Of eight patients Xpert MTB/RIF assay positive, seven died. Six deaths occurred in the hospital, and one died $\sim 1$ month after initiating anti-TB treatment. One TBM patient was already receiving treatment of disseminated TB when diagnosed with TBM and concurrent Cryptococcus (also EBV and JC positive), was readmitted for paradoxical CNS IRIS (TB and/or Cryptococcal-related), and ultimately survived 1 year. Five of the eight Xpert-positive patients also had concurrent EBV or JC present.

Among specimens run for Xpert MTB/RIF, CSF IFN- $\gamma$ levels were measured on $47 \mathrm{CSF}$ samples retrospectively (14 with cryptococcal meningitis, 33 without). The mean IFN- $\gamma$ level from 4 Xpert MTB/RIF positive samples was $4.05 \mathrm{IU} / \mathrm{mL}$ (95\% CI: 1.4 to 6.7 ). Of the remaining 43 samples, the mean IFN- $\gamma$ level was $1.45 \mathrm{IU} / \mathrm{mL}$ (95\% CI: 0.96 to 1.94$)$. Of the samples with cryptococcal meningitis, mean CSF IFN- $\gamma$ was $3.45 \mathrm{IU} / \mathrm{mL}$ (95\% CI: 0.78 to 6.12 ).

Finally, after performing CSF Cryptococcal antigen testing, CSF culture, AFB smear, Xpert MTB/RIF, along with extensive viral testing, there were $16.3 \%$ (43 of 264) with an abnormal CSF profile and clinical signs of meningitis without a microbiological diagnosis. Among these 43, the median CSF WBC was 45 cells/ $\mu \mathrm{L}$ (IQR, 4 to 153 ), with $100 \%$ lymphocytes (IQR, 81-100\%), and median CSF protein of $111 \mathrm{mg} / \mathrm{dL}$ (IQR, 71 to 227$)$. Of these $22(51 \%)$ were alive at discharge, $7(16 \%)$ were dead, and 14 (33\%) had unknown outcomes.

\section{DISCUSSION}

In this prospective cohort, after the rollout of antiretroviral therapy, we report that cryptococcal meningitis still causes the majority of meningitis among hospitalized adult patients in 2010-2012. Similar to three other recent publications, cryptococcal meningitis was more common than all other identified etiologies of meningitis combined. ${ }^{8-10}$ Thereafter, a confirmed diagnosis was difficult to ascertain. The prevalence of aseptic meningitis was $23 \%$ (71 of 314). Using intensive diagnostics for TB meningitis, we detected only 8 cases $(2.5 \%)$ of TB meningitis diagnosed by Xpert MTB/RIF assay. The AFB smear was negative in all of our samples. We also identified $1.6 \%$ cases of bacterial meningitis, $1 \%$ toxoplasmosis, and a number of patients with EBV, CMV, JC, or BK virus present in CSF. It is also possible that some of the unknown cases may have been caused by HIV infection itself. There appears to be little need for expansion of HSV PCR testing or intravenous acyclovir in our population. Although if further patients were tested, HSV likely would have been detected, the prevalence appears quite low and testing would not be cost-effective compared with other interventions. ${ }^{29}$ Overall, meningitis remains a highly prevalent condition among hospitalized HIV-infected adults in our setting, where HIV prevalence is estimated at $64 \%$ in hospitalized patients. ${ }^{30}$ Other settings that are similar with endemic TB, delayed HIV diagnosis, and challenges with retention-in-care likely also struggle with diagnosis and management of meningitis.

The recommended empiric use of ceftriaxone for meningitis should be reconsidered for patients with advanced HIV. ${ }^{2}$ Clearly bacterial meningitis is infrequent in our patient population, and in Zambia among those with HIV. ${ }^{8}$ In the general population in Malawi, Cryptococcus is nearly 2.3 times more common than all causes of bacterial meningitis combined in adults, ${ }^{9}$ with similar national epidemiology in South Africa. ${ }^{10}$
With these results, in both the general population and the population of inpatients with advanced HIV disease, and knowing that the cost of ceftriaxone $2 \mathrm{~g}$ (US\$2) is the same as the cryptococcal antigen lateral flow assay (CrAg LFA, \$2 Immy, Inc.), one might consider an algorithm for those HIV-infected patients initially presenting with meningitis, involving an initial cryptococcal antigen point-of-care test in the blood. If the CrAg LFA were positive, the physician would then measure CSF opening pressure, ${ }^{31}$ or perform a large volume lumbar puncture to reduce intracranial pressure. If cryptococcal antigen negative, one could do the lumbar puncture and perform gram stain, culture, and consider empiric ceftriaxone. Intensive TB diagnostics may be valuable in those who are cryptococcal antigen negative, including a chest radiograph, induced sputum, and/or further evaluation of lymphadenopathy. From a cost-effectiveness perspective, rapid expansion of CrAg LFA is a prudent public health investment.

Few studies have investigated the validity of Xpert MTB/ RIF in CSF specifically. ${ }^{32-34}$ The largest investigation thus far has been a South African cohort of 204 patients (87\% HIVinfected) with suspected meningitis. The Xpert MTB/RIF was found to be $67 \%$ sensitive and $94 \%$ specific when compared with PCR or culture confirmed TB meningitis, and was better than smear microscopy. ${ }^{23}$ Similar findings were described in a predominantly HIV-negative cohort in Vietnam with suspected TB meningitis, where Xpert MTB/RIF had a sensitivity of $59 \%$ compared with clinical diagnosis. ${ }^{24}$ Centrifuging the CSF should increase the sensitivity of the Xpert assay. ${ }^{23,35}$

Among 148 HIV-infected patients with suspected TBM in an Indian cohort, $2.1 \%$ of all CSF samples tested detected AFB by light-emitting diode auramine fluorescent microscopy, as compared with $24.6 \%$ of CSF samples that were Xpert MTB/RIF assay positive. ${ }^{36}$ In a retrospective Italian study, Xpert MTB/RIF detected 11 of 13 CSF samples that were culture positive, and an additional case that was culture negative but clinically presumed to be TB. ${ }^{37}$ Another Indian study reported excellent sensitivity and specificity of Xpert MTB/RIF in extrapulmonary samples, except for CSF, where sensitivity was only $29 \% .^{38}$

The role of complementary immunologic tests, such as CSF IFN- $\gamma$ levels is yet to be determined. In our samples, IFN- $\gamma$ levels were substantially higher in CSF that was positive by Xpert MTB/RIF - suggesting a possible role in the diagnosis of TB meningitis. Patel and others ${ }^{21}$ reported significantly higher IFN- $\gamma$ levels in the definite-TBM group than in the non-TBM group. The authors reported $92 \%$ sensitivity and $100 \%$ specificity in measuring IFN- $\gamma \geq 0.24 \mathrm{IU} / \mathrm{mL}$ in persons with negative Gram stain and cryptococcal antigen CSF against 48 non-TBM controls. ${ }^{21}$ Further prospective evaluation is warranted to determine the use of IFN- $\gamma$ in those with suspected TB meningitis after exclusion of Cryptococcus; both of which illicit a type-1 helper T cell (Th1) immune response.

Finally, we identified EBV in $42 \%$ of patients tested; many with EBV alone and others co-infected with Cryptococcus, $\mathrm{TB}$, or other viral pathogens. Similar findings have been confirmed in a Zambian cohort with advanced HIV, where $28 \%$ of CSF samples had EBV detected by PCR, ${ }^{8}$ and in two additional studies from Malawi. ${ }^{39,40}$ Of note, one of the studies from Malawi described a prevalence of $61 \%$ among those with HIV and bacterial meningitis and found EBV to be associated with HIV infection, and EBV viral load to be associated with increased mortality. ${ }^{39}$ Kelly and others speculated 
that EBV might be a non-pathogenic marker of immunosuppression, being shed from activated B-cells trafficking into the CSF during meningitis. Conversely, an alternative view would be that EBV infection is the primary insult, which predisposes severely immunocompromised patients to other forms of meningitis. The CNS lymphoma may also be associated with EBV in the CSF, though further diagnostics for evaluation are lacking in our setting.

Another study out of Malawi evaluated viral etiologies of aseptic meningitis, and found a very small proportion of patients with aseptic meningitis $(8 \%) .{ }^{40}$ Of these, $25 \%$ were EBV positive in the CSF, all of whom were HIV-infected. Further investigation into the significance of EBV in the CSF is needed. Quantitative values from the CSF and serum may be helpful to distinguish those with CNS lymphoma from those with non-pathogenic presence of EBV.

Several limitations of the current cohort and setting are worth mentioning. First, as the main focus of the study was to evaluate the etiology of meningitis, valuable clinical results such as chest radiograph results, response to empiric therapies, and clinical outcomes were incomplete. The local laboratory was unable to provide Mycobacteria culture or CSF glucose. Ideally TB culture, though not clinically timely, would provide a gold standard by which to compare the Xpert MTB/RIF assay. In 2013, we began processing CSF glucose using a handheld glucometer. Additionally, CSF VDRL is an insensitive diagnostic test for syphilis. Thus, a negative value does not rule out a diagnosis of syphilis. Serum RPR may have been more informative.

In summary, we identified a microbiologic diagnosis for clinical meningitis in a cohort of HIV-infected Ugandan inpatients. The most common etiology was cryptococcal meningitis. Among persons negative for Cryptococcus, the Xpert MTB/ RIF assay was of moderate yield ( $22 \%$ prevalence) in the CSF (2.5\% overall prevalence). Forty-two percent of CSF samples tested were EBV positive, though the significance of this requires additional quantitative investigation. Exploration of new $\mathrm{TB}$ diagnostics along with diagnostic algorithms for evaluation of meningitis in resource-limited settings remains critical.

Received July 21, 2014. Accepted for publication September 19, 2014.

Published online November 10, 2014.

Note: Supplemental Methods appear at www.ajtmh.org.

Financial support: Research support for aspects of this project was received from the National Institutes of Health and Fogarty International Center (R21NS065713, K23AI073192, U01AI089244, T32AI055433, R25TW009345, T32HD068229). Partially funded by an Interagency Agreement between CDC and the Defense Threat Reduction Agency.

Disclaimer: The findings and conclusions in this document are those of the authors and do not necessarily represent the views of the Centers for Diseases Control and Prevention or Minnesota Department of Health.

Authors' addresses: Radha Rajasingham, University of Minnesota, Infectious Diseases and International Medicine, Minneapolis, MN, and Infectious Diseases Institute, Makarere University, Kampala, Uganda, E-mail: radha.rajasingham@gmail.com. Joshua Rhein and Darlisha A. Williams, University of Minnesota, Medicine, Minneapolis, MN, and Infectious Diseases Institute, Makarere University, Kampala, Uganda, E-mails: joshua.rhein@gmail.com and coat.trial@gmail.com. Kate Klammer, Minnesota Department of Health, N/A, St. Paul, MN, E-mail: kate.klammer@mn.state.us. Abdu Musubire, Henry Nabeta, and Andrew Akampurira, Infectious Diseases Institute, Makarere University, Kampala, Uganda, E-mails: amusubire@yahoo.com, hwnabeta@ gmail.com, and scandrie@yahoo.com. Eric C. Mossel and Barry R. Miller, Centers for Disease Control and Prevention, N/A, Fort Collins, $\mathrm{CO}$, and Centers for Disease Control and Prevention/Fort Collins, Division of Vector-Borne Infectious Diseases, Fort Collins, CO, E-mails: ilv8@cdc.gov and brm4@cdc.gov. Dave J. Boxrud, Minnesota Department of Health, N/A, St Paul, MN, E-mail: dave.boxrud@state.mn.us. Melissa A. Rolfes and Supatida Tengsupakul, University of Minnesota, Medicine, Minneapolis, MN, E-mails: melissa.rolfes@gmail.com and tengs001@umn.edu. Alfred O. Andama, Makerere University College of Health Sciences, Department of Medicine, Kampala, Uganda, E-mail: andama.alf@gmail.com. David B. Meya, Makerere University, Faculty of Medicine, Kampala, Uganda, E-mail: david.meya@gmail .com. David R. Boulware, University of Minnesota, Medicine, Minneapolis, MN, boulw001@umn.edu.

\section{REFERENCES}

1. WHO, 2013. Health Topics: Meningitis. Available at: http://www .who.int/topics/meningitis/en/. Accessed January 20, 2013.

2. WHO, 2007. Standardized Treatment of Bacterial Meningitis in Africa in Epidemic and Non-Epidemic Situations. WHO/CDS/ EPR/2007.3. Geneva: WHO Press.

3. Jarvis JN, Meintjes G, Williams A, Brown Y, Crede T, Harrison TS, 2010. Adult meningitis in a setting of high HIV and TB prevalence: findings from 4961 suspected cases. BMC Infect Dis 10: 67.

4. Hakim JG, Gangaidzo IT, Heyderman RS, Mielke J, Mushangi E, Taziwa A, Robertson VJ, Musvaire P, Mason PR, 2000. Impact of HIV infection on meningitis in Harare, Zimbabwe: a prospective study of 406 predominantly adult patients. AIDS 14: $1401-1407$.

5. Amuron B, Levin J, Birunghi J, Namara G, Coutinho A, Grosskurth H, Jaffar S, 2011. Mortality in an antiretroviral therapy programme in Jinja, south-east Uganda: a prospective cohort study. AIDS Res Ther 8: 39.

6. Bhagwan S, Naidoo K, 2011. Aetiology, clinical presentation, and outcome of meningitis in patients coinfected with human immunodeficiency virus and tuberculosis. Aids Res Treat 2011: 180352.

7. Cohen DB, Zijlstra EE, Mukaka M, Reiss M, Kamphambale S, Scholing M, Waitt PI, Neuhann F, 2010. Diagnosis of cryptococcal and tuberculous meningitis in a resource-limited African setting. Trop Med Int Health 15: 910-917.

8. Siddiqi OK, Ghebremichael M, Dang X, Atadzhanov M, Kaonga P, Khoury MN, Koralnik IJ, 2014. Molecular diagnosis of central nervous system opportunistic infections in HIV-infected Zambian adults. Clin Infect Dis 58: 1771-1777.

9. Wall EC, Everett DB, Mukaka M, Bar-Zeev N, Feasey N, Jahn A, Moore M, van Oosterhout JJ, Pensalo P, Baguimira K, Gordon SB, Molyneux EM, Carrol ED, French N, Molyneux ME, Heyderman RS, 2014. Bacterial meningitis in Malawian adults, adolescents, and children during the era of antiretroviral scale-up and Haemophilus influenzae type b vaccination, 20002012. Clin Infect Dis 58: e137-e145.

10. National Institute for Communicable Diseases, 2012. GERMS-SA Annual Report 2012. Meiring S, Quan V, Govender N, Crowther-Gibson P, eds. Available at: http://www.nicd.ac.za/? page $=$ publications\&id $=155$. Accessed October 28, 2014 .

11. Berhe T, Melkamu Y, Amare A, 2012. The pattern and predictors of mortality of HIV/AIDS patients with neurologic manifestation in Ethiopia: a retrospective study. AIDS Res Ther 9: 11.

12. Alemu AS, Kempker RR, Tenna A, Smitson C, Berhe N, Fekade D, Blumberg HM, Aseffa A, 2013. High prevalence of cryptococcal antigenemia among HIV-infected patients receiving antiretroviral therapy in Ethiopia. PLoS ONE 8: e58377.

13. Beyene T, Woldeamanuel Y, Asrat D, Ayana G, Boulware DR, 2013. Comparison of cryptococcal antigenemia between antiretroviral naïve and antiretroviral experienced HIV positive patients at two hospitals in Ethiopia. PLoS ONE 8: e75585.

14. Afifi S, Karsany MS, Wasfy M, Pimentel G, Marfin A, Hajjeh R, 2009. Laboratory-based surveillance for patients with acute meningitis in Sudan, 2004-2005. Eur J Clin Microbiol Infect Dis 28: $429-435$. 
15. Trachtenberg JD, Kambugu AD, McKellar M, Semitala F, Mayanja-Kizza H, Samore MH, Ronald A, Sande MA, 2007. The medical management of central nervous system infections in Uganda and the potential impact of an algorithm-based approach to improve outcomes. Int J Infect Dis 11: 524-530.

16. Torok ME, Chau TT, Mai PP, Phong ND, Dung NT, Chuong LV, Lee SJ, Caws M, de Jong MD, Hien TT, Farrar JJ, 2008. Clinical and microbiological features of HIV-associated tuberculous meningitis in Vietnamese adults. PLoS One 3: e1772.

17. Kennedy DH, Fallon RJ, 1979. Tuberculous meningitis. JAMA 241: 264-268.

18. Caws M, Thwaites GE, Duy PM, Tho DQ, Lan NT, Hoa DV, Chau TT, Huyen MN, Anh PT, Chau NV, Chinh TN, Stepniewska K, Farrar J, 2007. Molecular analysis of Mycobacterium tuberculosis causing multidrug-resistant tuberculosis meningitis. Int J Tuberc Lung Dis 11: 202-208.

19. Thwaites GE, Chau TT, Stepniewska K, Phu NH, Chuong LV, Sinh DX, White NJ, Parry CM, Farrar JJ, 2002. Diagnosis of adult tuberculous meningitis by use of clinical and laboratory features. Lancet 360: 1287-1292.

20. Juan RS, Sanchez-Suarez C, Rebollo MJ, Folgueira D, Palenque E, Ortuno B, Lumbreras C, Aguado JM, 2006. Interferon gamma quantification in cerebrospinal fluid compared with PCR for the diagnosis of tuberculous meningitis. J Neurol 253: 1323-1330.

21. Patel VB, Singh R, Connolly C, Kasprowicz V, Ndung'u T, Dheda K, 2011. Comparative utility of cytokine levels and quantitative RD-1-specific $\mathrm{T}$ cell responses for rapid immunodiagnosis of tuberculous meningitis. J Clin Microbiol 49: 3971-3976.

22. O'Grady J, Bates M, Chilukutu L, Mzyece J, Cheelo B, Chilufya M, Mukonda L, Mumba M, Tembo J, Chomba M, Kapata N, Maeurer M, Rachow A, Clowes P, Hoelscher M, Mwaba P, Zumla A, 2012. Evaluation of the Xpert MTB/RIF assay at a tertiary care referral hospital in a setting where tuberculosis and HIV infection are highly endemic. Clin Infect Dis 55: $1171-1178$.

23. Patel VB, Theron G, Lenders L, Matinyena B, Connolly C, Singh R, Coovadia Y, Ndung'u T, Dheda K, 2013. Diagnostic accuracy of quantitative PCR (Xpert MTB/RIF) for tuberculous meningitis in a high burden setting: a prospective study. PLoS Med 10: e1001536.

24. Nhu NT, Heemskerk D, Thu do DA, Chau TT, Mai NT, Nghia HD, Loc PP, Merson L, Thinh TT, Day J, Chau N, Wolbers M, Farrar J, Caws M, 2014. Evaluation of GeneXpert MTB/RIF for diagnosis of tuberculous meningitis. J Clin Microbiol 52: $226-233$

25. Boulware DR, Meya DB, Muzoora C, Rolfes MA, Huppler Hullsiek K, Musubire A, Taseera K, Nabeta HW, Schutz C, Williams DA, Rajasingham R, Rhein J, Thienemann F, Lo MW, Nielsen K, Bergemann TL, Kambugu A, Manabe YC, Janoff EN, Bohjanen PR, Meintjes G, 2014. Timing of antiretroviral therapy after diagnosis of cryptococcal meningitis. $N$ Engl J Med 370: 2487-2498.

26. Bahr N, Boulware DR, Marais S, Scriven J, Wilkinson RJ, Meintjes G, 2013. Central nervous system immune reconstitution inflammatory syndrome. Curr Infect Dis Rep 15: 583-593.

27. Boulware DR, Rolfes MA, Rajasingham R, von Hohenberg M, Qin Z, Taseera K, Schutz C, Kwizera R, Butler EK, Meintjes G,
Muzoora C, Bischof JC, Meya DB, 2014. Multisite validation of cryptococcal antigen lateral flow assay and quantification by laser thermal contrast. Emerg Infect Dis 20: 45-53.

28. Blakemore R, Story E, Helb D, Kop J, Banada P, Owens MR, Chakravorty S, Jones M, Alland D, 2010. Evaluation of the analytical performance of the Xpert MTB/RIF assay. J Clin Microbiol 48: 2495-2501.

29. Rajasingham R, Rolfes MA, Birkenkamp KE, Meya DB, Boulware DR, 2012. Cryptococcal meningitis treatment strategies in resource-limited settings: a cost-effectiveness analysis. PLoS Med 9: e1001316.

30. Wanyenze R, Kamya M, Liechty CA, Ronald A, Guzman DJ, Wabwire-Mangen F, Mayanja-Kizza H, Bangsberg DR, 2006. HIV counseling and testing practices at an urban hospital in Kampala, Uganda. AIDS Behav 10: 361-367.

31. Meda J, Kalluvya S, Downs JA, Chofle AA, Seni J, Kidenya B, Fitzgerald DW, Peck RN, 2014. Cryptococcal meningitis management in Tanzania with strict schedule of serial lumber punctures using intravenous tubing sets: an operational research study. J Acquir Immune Defic Syndr 66: e31-e36.

32. Hillemann D, Rusch-Gerdes S, Boehme C, Richter E, 2011. Rapid molecular detection of extrapulmonary tuberculosis by the automated GeneXpert MTB/RIF system. J Clin Microbiol 49: $1202-1205$

33. Zeka AN, Tasbakan S, Cavusoglu C, 2011. Evaluation of the GeneXpert MTB/RIF assay for rapid diagnosis of tuberculosis and detection of rifampin resistance in pulmonary and extrapulmonary specimens. J Clin Microbiol 49: 4138-4141.

34. Causse M, Ruiz P, Gutierrez-Aroca JB, Casal M, 2011. Comparison of two molecular methods for rapid diagnosis of extrapulmonary tuberculosis. J Clin Microbiol 49: 3065-3067.

35. Boulware DR, 2013. Utility of the Xpert MTB/RIF assay for diagnosis of tuberculous meningitis. PLoS Med 10: e1001537.

36. Alvarez-Uria G, Azcona JM, Midde M, Naik PK, Reddy S, Reddy R, 2012. Rapid diagnosis of pulmonary and extrapulmonary tuberculosis in HIV-infected patients. comparison of LED fluorescent microscopy and the GeneXpert MTB/RIF assay in a district hospital in India. Tuberc Res Treat 2012: 932862.

37. Tortoli E, Russo C, Piersimoni C, Mazzola E, Dal Monte P, Pascarella M, Borroni E, Mondo A, Piana F, Scarparo C, Coltella L, Lombardi G, Cirillo DM, 2012. Clinical validation of Xpert MTB/RIF for the diagnosis of extrapulmonary tuberculosis. Eur Respir J 40: 442-447.

38. Vadwai V, Boehme C, Nabeta P, Shetty A, Alland D, Rodrigues C, 2011. Xpert MTB/RIF: a new pillar in diagnosis of extrapulmonary tuberculosis? J Clin Microbiol 49: 2540-2545.

39. Kelly MJ, Benjamin LA, Cartwright K, Ajdukiewicz KM, Cohen DB, Menyere M, Galbraith S, Guiver M, Neuhann F, Solomon T, Lalloo DG, Heyderman RS, 2012. Epstein-Barr virus coinfection in cerebrospinal fluid is associated with increased mortality in Malawian adults with bacterial meningitis. $J$ Infect Dis 205: 106-110.

40. Benjamin LA, Kelly M, Cohen D, Neuhann F, Galbraith S, Mallewa M, Hopkins M, Hart IJ, Guiver M, Lalloo DG, Heyderman RS, Solomon T, 2013. Detection of herpes viruses in the cerebrospinal fluid of adults with suspected viral meningitis in Malawi. Infection 41: 27-31. 